\title{
A COMPACT X-RAY SOURCE BASED ON COMPTON SCATTERING
}

\author{
E. Bulyak, A. Dovbnya, P. Gladkikh, Yu. Grigor'ev, I. Karnaukhov, A. Khodyachikh, \\ S. Kononenko, V. Lapshin, V. Molodkin, A. Mytsykov, V. Nemoshkalenko, A. Shcherbakov, \\ A. Shpak, Yu. Telegin, A., Zelinsky \\ NSC KIPT, 310108, Kharkov, Ukraine
}

\begin{abstract}
The paper discusses the use of Compton backscattering of an intense laser light on a relativistic electron beam to produce high-intensity $\gamma$ beams, and also the practicability of the concept. Requirements on the parameters of electron and photon beams are described, and the lattice of the electron storage ring with a beam energy up to 150 $\mathrm{MeV}$ is proposed, wherein experiments on intense photon beam generation and laser cooling of the electron beam can be conducted. Electron beam parameters in the storage ring are calculated with due regard for the intrabeam scattering, photon beam parameters are also estimated. The scheme of experiment that enables the production of a photon beam with an intensity of $\sim 10^{13} \mathrm{~s}^{-1}$ is proposed.
\end{abstract}

\section{INTRODUCTION}

Telnov [1] has proposed the method of cooling the electron beam with a laser light, that makes it possible to reduce the transverse beam emittance by 1 to 3 orders of magnitude.

Huang and Ruth [2] have put forward a proposal to create a laser-electron storage ring (LESR) with an electron energy ranging from a few $\mathrm{MeV}$ to several hundred $\mathrm{MeV}$, wherein the laser cooling is used for compensating the intrabeam scattering effects; this permits one to minimize transverse beam emittances and to produce intense beams of photons.

The electron beam is injected to the storage ring, and simultaneously, an intense laser pulse is introduced in a high - $Q$-factor optical resonator. The laser pulse path length is chosen in such a way as to exactly correspond to the revolution period of electron beam. Then, with each revolution, electrons collide with a light pulse in the resonator focus. The laser pulse in the LESR acts on the beam much like a very strong undulator or a wiggler, therefore a rapid laser cooling leads to very low beam emittances even at moderate (about $100 \mathrm{MeV}$ ) energies.

\section{CHOICE OF THE LESR LATTICE}

In deciding on a particular lattice of the LESR intended for performing experiments on laser cooling of the stored beam and on $\gamma$ generation with the use of Compton backscattering, it is necessary to solve the following problems:

- to provide the maximum density of the electron beam in the region of its interaction with the photon beam (interaction point - IP) stored in the optical resonator;

- an increase in the electron beam density leads to an increase in the nonlinear Coulomb tune shift and in the enhancement of intrabeam scattering effects; therefore, the beam dimensions around the whole circumference of the ring must be much greater than at the IP. In other words, the lattice must comprise only one low- $\beta$ insertion;

- the strong focusing of the beam in the low- $\beta$ insertion determines a high natural chromaticity of the ring. The presence of only one low- $\beta$ insertion reduces the degree of storage ring symmetry down to $N=1$ and this drastically extends the spectrum of azimuthal harmonics of nonlinear perturbations and restricts the dynamic aperture (DA).

In view of these considerations, we have suggested a version for reconstruction of the operative storage ring $\mathrm{N}$ 100 [3] in order to carry out Compton backscattering experiments. The layout of the focusing structure elements are shown schematically in fig. 1.

The arc lattice and phase advances of betatron oscillations are chosen proceeding from the requirements of providing optimum conditions for correction of natural chromaticity of the ring. The matching of arcs and long straight sections is accomplished by quadrupole lens triplets. The existing bending magnets of the ring $\mathrm{N}-100$ with a bending angle $\varphi=90^{\circ}$ will be used.

The momentum compaction factor under operating conditions with two dispersion-free long straight sections will be high. The equilibrium energy spread during Compton photon generation makes up several percent [2] and holding such a beam in the facility with a high momentum compaction factor calls for great voltages of $R F$ power supply. The placement of a sufficient number of accelerating structures in the compact facility appears impossible.

The abandonment of the dispersion-free injection section makes the solution of this problem possible. In this case, the mode of operation of the storage ring can be easily varied to control the value of the momentum compaction factor. At these operating conditions, the long straight section with the IP remains dispersion-free, while at the injection section the dispersion function is non-zero. 
Retuning is realized by all quadrupole lenses of the ring, while the tunes remain unchanged and the optical functions of the ring vary insignificantly (naturally, exclusive of the dispersion function). Among the advantages of this mode of operation is the fact that the existence of an additional dispersion section makes it possible to optimize the task of correcting the natural chromaticity of the ring, this being a currently central problem for compact rings with great beam energy spreads. With the additional high-dispersion section it appears possible to weaken the intrabeam scattering effects and to diminish the electron beam dimensions that have set in. Since retuning is carried out at fixed tunes, the beam can be stored at operating conditions with a dispersion-free injection section, and thereafter the operating conditions with a low momentum compaction factor can be adjusted. Incidentally, the DA of the storage ring permits beam injection at conditions with a lower value of momentum compaction factor, too.

The main parameters of the storage ring for the stored beam energy $E=100 \mathrm{MeV}$ are given in table 1 (values given in brackets correspond to a low momentum compaction factor). The beam parameters with due regard for the intrabeam scattering effects were calculated using the results of [4]. In the calculations, the coupling coefficient of vertical and radial betatron oscillations was put to be 0.1. The stored current in the bunch was assumed to be $10 \mathrm{~mA}\left(\sim 2.5 \cdot 10^{9}\right.$ particles).

Table 1.

\begin{tabular}{l|c}
\multicolumn{2}{c}{ Main parameters of the storage ring } \\
\hline \multicolumn{1}{c}{ Parameter } & Value \\
Operating energy range $[\mathrm{MeV}]$ & $40-160$ \\
Injection energy [MeV] & 70 \\
Circumference [m] & 12.004 \\
Tunes: $\quad$ horizontal, $Q_{x}$ & 3.162 \\
\multicolumn{1}{c}{$\quad$ vertical, $Q_{z}$} & 1.220 \\
$\quad$ Amplitude functions at IP [cm]: & 3 \\
$\quad$ horizontal, $\beta_{x}$ & 6 \\
$\quad$ vertical, $\beta_{z}$ & 0.4 \\
$R F$ voltage amplitude [MV] & 699.28 \\
$R F$ frequency [MHz] & 28 \\
Harmonic number, $h$ & 0.098 \\
Momentum compaction factor, $\alpha$ & $(0.0061)$ \\
& 0.041 \\
Synchrotron oscillation tune, $Q_{s}$ & $(0.010)$ \\
Energy acceptance, [\%] & $\pm 3( \pm 12)$ \\
Natural emittance $\varepsilon_{x},[\mathrm{~nm}]$ & $14.7(14.0)$ \\
Horizontal beam size at $I P$ with $I B S,[\mu m]$ & $120(160)$ \\
Natural chromaticity: & \\
$\quad$ in horizontal plane, $\xi_{x}$ & $-3.89(-4.22)$ \\
$\quad$ in vertical plane, $\xi_{z}$ & $-10.9(-10.9)$ \\
\hline
\end{tabular}

Fig. 2 shows the amplitude and dispersion functions of focusing for the operating conditions with low momentum compaction factor $\alpha=0.0061$.
The DA dimensions calculated for peak values of amplitude functions are found to be about $\pm 22 \mathrm{~mm}$ and $\pm 30 \mathrm{~mm}$ in horizontal and vertical planes, respectively (energy spread $\delta= \pm 0.5 \%$ ). The DA dimensions in the horizontal plane are approximately coincident with the physical aperture size, while in the vertical plane they are far in excess of the latter, and are quite sufficient for realizing injection, beam storage and conducting physical experiments. The problems of correcting natural chromaticity of the ring for high values of the steady energy spread need further consideration.

\section{OPTICAL EQUIPMENT OF THE LESR}

The straight section of the storage ring and the half-axis of the optical resonator are aligned. The resonator is formed by two high-quality mirrors and with the reflection coefficients close to unity. The doubled spacing between the resonator mirrors is equal to the electron orbit length in the storage ring. The single-mode laser generates light pulses of $50 \mathrm{~ns}$ length (light bunch length $\sigma_{f}=15 \mathrm{~mm}$ ), with a pulse-repetition frequency $f \sim 10^{4}$ and an average power $P=100 \mathrm{~W}$ (flash energy being about 10 $m J)$. The laser active element is manufactured from an yttrium-aluminum garnet crystal activated with neodymium $(N d: Y A G)$. The wavelength of the main harmonic is $\lambda=1.06 \mu \mathrm{m}$. The photon beam is focused so that its minimum transverse size is achieved in the middle of the interaction region.

\section{PARAMETERS OF ELECTRON AND PHOTON BEAMS}

Proceeding from the parameters of storage ring and optical equipment as given in the previous sections, as well as from the consideration presented in [2], we can estimate the parameters of the electron beam and the photon beam in the LESR N-100 for the electron beam energy $E=100 \mathrm{MeV}$, the photon energy in the beam $\varepsilon_{\gamma_{0}}=$ $1.17 \mathrm{eV}$ (this corresponding to the laser wavelength of $1.06 \mu m$ - Nd: YAG laser).

For the given laser flash energy $E_{L}=10 \mathrm{~mJ}$, the number of photons in the flash will be $n_{\gamma}=5.33 \cdot 10^{16}$. The average energy loss by the electron on its interaction with the laser flash photon is $\varepsilon_{\gamma} \sim 100 \mathrm{KeV}$, and the average energy losses by the electron beam per turn are given by

$(\Delta E)_{e}=\varepsilon_{\gamma} \frac{n_{\gamma} \sigma_{c}}{s}=\varepsilon_{\gamma} \frac{n_{\gamma} \sigma_{c}}{\pi \sigma_{x}^{2}} \approx 5 \mathrm{eV}$,

where $\sigma_{c}$-Compton scattering cross-section,

$\sigma_{x}$ - horizontal size of electron beam.

At a stored beam energy $E=100 \mathrm{MeV}$, the synchrotron radiation losses make up $17.7 \mathrm{eV}$. In principle, this will permit experimental verification of laser cooling effects. The balance between the damping rate and the growth rate of transverse emittance gives the values of minimum normalized transverse emittances: 
$\left(\varepsilon_{x}^{n}\right)_{\min }=\frac{3}{10} \frac{\lambda_{C}}{\lambda_{L}} \beta_{x}^{*} \approx 1.8 \cdot 10^{-8}$

where $\beta_{x}^{*}$ - amplitude function in $I P$.

At Compton scattering the energy spread in the beam increases because of energy fluctuations of scattered electrons. The energy spread that set in is given by:

$\left(\sigma_{\delta}\right)_{\min } \equiv\left(\frac{\sigma_{E}}{E}\right)_{\min }=\sqrt{\frac{7}{5} \frac{\lambda_{C}}{\lambda_{L}} \gamma} \approx 2.6 \%$

For the RF acceptance $\sigma_{R F}=12 \%$ the quantum lifetime will be $\tau_{q} \sim 450 \mathrm{~s}$, this being quite sufficient for conducting experiments.

At a particle number in the bunch $N_{e} \sim 2.5 \cdot 10^{9}$, a mirror reflectivity of 0.9999 , the beam size at the IP $\sigma_{x} \sim 150 \mu \mathrm{m}$ and the laser pulse frequency $f=10^{4}$, the total number of Compton photons will make up $N_{\gamma} \sim 2.5 \cdot 10^{12} \mathrm{~s}^{-1}$. An increase in the energy spread of the electron beam as it interacts with the laser photon beam will depress the intrabeam scattering effects, and this must cause the Compton photon yield to increase.

\section{CONCLUSION}

A scheme of re-designing the electron storage ring N-100 to generate backward Compton gamma-quanta with an intensity of $\sim 10^{13} s^{-1}$ is proposed. The facility can provide high-intensity $\gamma$ beams over a wide energy range due to both the variation in the wavelength of laser light and energy readjustment of the electron storage ring. The facility will permit research into the process of laser cooling of the electron beam in the storage ring.

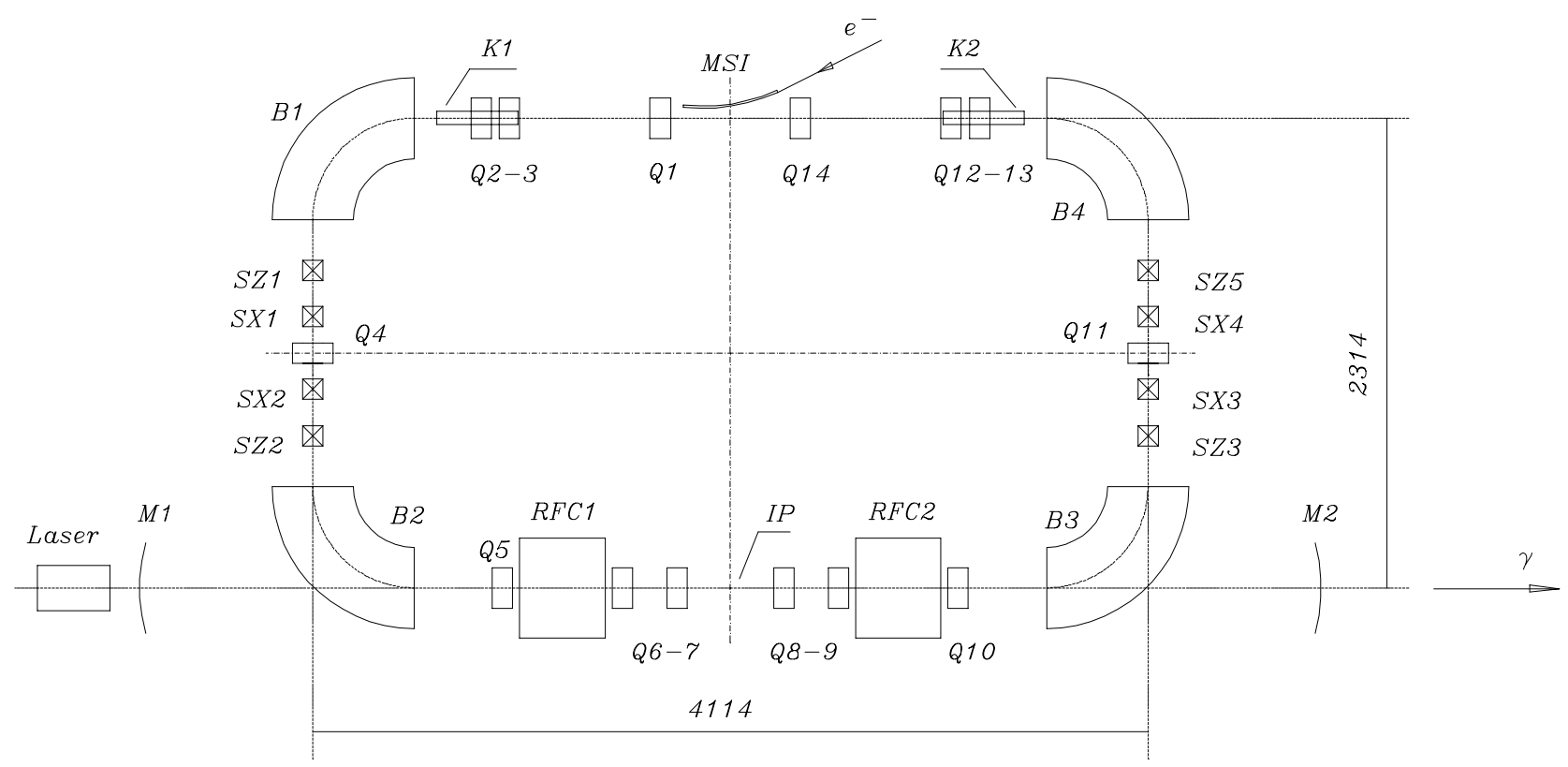

Fig. 1. Layout of the storage ring.

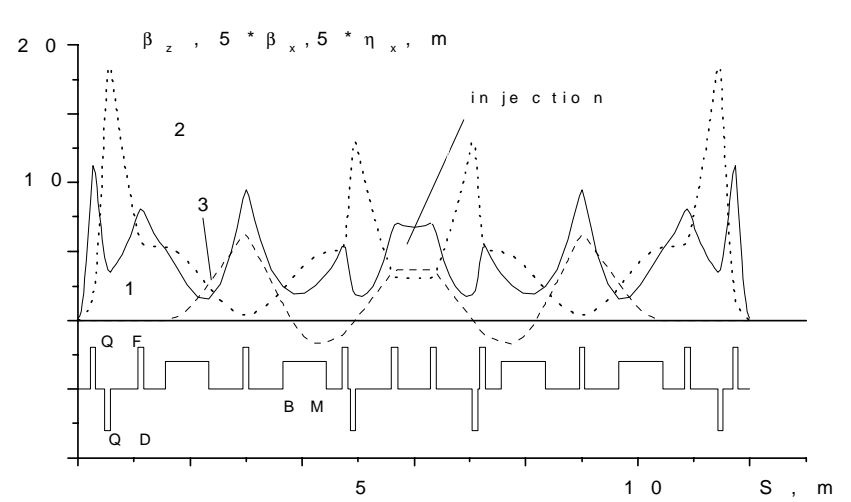

\section{REFERENCES}

[1] V. Telnov, Phys. Rev. Letters, 78, 1997, p.4757

[2] Z. Huang, R. Ruth, SLAC-PUB-7556, September 1997, p.11.

[3] Yu.N. Grigoriev et al., At. Ehnerg. 23(6), 1967, p.531

[4] J.D.Bjorken and S.K.Mtingwa, Intra-beam scattering, Part.Accel. 13, 1983, p.115.

Figure 2. Amplitude functions (horizontal (1), vertical (2) and dispersion(3)) of focusing for the mode of operation with a momentum compaction factor $(\alpha=0.0061)$. BM bending magnets, $Q F$ and $Q D$ - quadrupole lenses focusing and defocusing in the horizontal plane. 